\title{
The Removal Method of the Blasting Vibration Signal Trend Item and Noise
}

\author{
Hailong Wang, ${ }^{1}$ Haobo Bai $\left(\mathbb{D},{ }^{2}\right.$ Yan Zhao $\left(\mathbb{D},{ }^{3}\right.$ Dongsheng Wang, ${ }^{3}$ Xiaojing Wang, ${ }^{4}$ \\ and Shenghua Wang ${ }^{4}$ \\ ${ }^{1}$ Hebei University of Architecture, Zhangjiakou 075000, China \\ ${ }^{2}$ Hebei Key Laboratory of Diagnosis, Reconstruction and Anti-Disaster of Civil Engineering, \\ Hebei Innovation Center of Transportation Infrastructure in Cold Region, Zhangjiakou 075000, China \\ ${ }^{3}$ School of Mechanics and Civil Engineering, China University of Mining and Technology (Beijing), Beijing 100083, China \\ ${ }^{4}$ Beiwang Construction Group Co Ltd, Chengde 067000, China \\ Correspondence should be addressed to Haobo Bai; 915624065@qq.com and Yan Zhao; 304965624@qq.com
}

Received 15 September 2021; Accepted 11 October 2021; Published 23 October 2021

Academic Editor: Fan Deyuan

Copyright (c) 2021 Hailong Wang et al. This is an open access article distributed under the Creative Commons Attribution License, which permits unrestricted use, distribution, and reproduction in any medium, provided the original work is properly cited.

\begin{abstract}
Aiming at the problem that the blasting vibration signal contains trend items and noise interference, a signal processing method based on the Fourier decomposition method (FDM) is introduced. Firstly, based on the FDM theory, the original blasting vibration signal is decomposed to obtain several modal components; then, the dominant component of the signal is filtered according to the dominant frequency of the modal component and its correlation with the original signal; finally, the dominant component is reconstructed to remove the trend item and noise at the same time; spectrum analysis is carried out to verify the effectiveness of the method. The results show that FDM can effectively disperse low-frequency trend items, high-frequency noise, and useful signal information while decomposing signals containing trend terms and noise; compared with the existing common methods, it can more effectively retain the original information, which provides a reference for the trend item and noise removal of similar blasting vibration signals.
\end{abstract}

\section{Introduction}

While constructing mountainous tunnels using drilling and blasting methods, there will be a destructive impact on the surrounding environment, so controlling the hazards caused by blasting is particularly critical. Moreover, extracting the detailed characteristic information contained in the blasting vibration signal is of great significance for controlling the influence of blasting vibration and optimizing blasting parameters. However, during signal acquisition, under the effect of the installation of the instrument and the surrounding environment, there may exist trend items in the signal near the blasting area, and high-frequency noise may be induced in the signal due to the influence of other process construction. The existence of trend items and noise is extremely unfavourable for extracting detailed features of blasting signals.
Due to the abrupt and instantaneous nature of blasting, the blasting vibration signal appears as a typical nonlinear and nonstationary signal. To deal with this type of signal problem, a wide range of analysis methods, including the EMD (empirical mode decomposition) method [1], EEMD (ensemble empirical mode decomposition) method [2], CEEMD (complete ensemble empirical mode decomposition) method [3], CEEMDAN (complete ensemble empirical mode decomposition with adaptive noise) method [4], and VMD (variational mode decomposition) method [5], were developed.

While using the EMD method to perform signal decomposition, end-point oscillation and modal aliasing may occur. In order to extract the detailed features of the signal more accurately, the EEMD method [6] and the VMD method [7] have been successively used in the removal of the trend term of the blasting vibration signal. For the problem 
of high-frequency noise interference in the signal, the CEEMDAN method [8], the wavelet threshold method [9], and the wavelet packet threshold method [10] are widely adopted.

In recent years, simultaneously removing the trend items and noise interference contained in blasting vibration signals has become a research hotspot. By combining the EEMD method with wavelet analysis, Li et al. [11] took the frequency band distribution as the criterion to remove the trend item in the blasting vibration signal and used the wavelet threshold method for denoising. Fu et al. [12] used the CEEMD method and the sparse baseline estimation denoising method to remove the trend term in the signal near the blasting area and then used the hidden Markov model to denoise the signal.

However, the EMD method and its improved method are able to fundamentally solve the problem of modal aliasing while decomposing the signal, which is unfavourable to the extraction of signal feature information. The wavelet threshold method and wavelet packet threshold method are limited by the choice of wavelet basis function and the number of decomposition levels in signal processing.

The Fourier decomposition method (FDM) has been widely used in the signal analysis in recent years [13-15]. Because it is based on the Fourier transform, the decomposition shows orthogonality, completeness, and selfadaptability, effectively eliminating the modal aliasing effect produced by traditional methods. This method has unique advantages for analyzing nonlinear and nonstationary blasting vibration signals.

Therefore, this paper introduces an FDM-based method of removing blasting vibration signal trend item and noise. Firstly, FDM is used to decompose the original signal into several modal components. Then, the trend item is filtered out and eliminated by the dominant frequency information of the modal component, and the dominant component containing the useful information of the signal is filtered by the cross-correlation coefficient. Finally, the dominant component obtained from the screening is reconstructed to obtain a pure signal.

\section{FDM Time-Frequency Analysis Theory}

2.1. FDM Theory Explanation. Singh et al. [16] proposed a new signal decomposition method based on Fourier transform (FDM), which can be used to analyze nonlinear and nonstationary signals; this method can adaptively search and analyze Fourier intrinsic frequency band functions (AFIBFs) in the Fourier domain; thus, a series of Fourier intrinsic frequency band functions and a residual component can be obtained, obtaining multicomponent signals as the only representation of constant and single-component signals. The mathematical model can be expressed by the following formula:

$$
x(t)=\sum_{i=1}^{M} y_{i}(t)+n(t),
$$

where $n(t)$ is the residual component and $y_{i}(t) \in C^{\infty}[a, b]$ is the Fourier intrinsic frequency band function (FIBF). FIBFs have the properties expressed as follows: the functions are all zero-mean functions, and different component functions are orthogonal to each other; the instantaneous frequency and instantaneous amplitude of the analytical functions provided by FIBFs are not less than 0 .

$$
\begin{aligned}
\int_{a}^{b} y_{i}(t) \mathrm{d} t & =0, \\
\int_{a}^{b} y_{i}(t) y_{j}(t) \mathrm{d} t & =0(i \neq j), \\
y_{i}(t)+j \hat{y}_{i}(t) & =a_{i}(t) \exp \left(j \phi_{i}(t)\right) ; \quad \forall t, a_{i}(t), \frac{\mathrm{d}}{\mathrm{d} t} \phi_{i}(t) \geq 0 .
\end{aligned}
$$

It can be known that the FDM method has the characteristics of completeness, orthogonality, locality, and selfadaptability. After Fourier decomposition of the signal, the useful information in the signal can be extracted from the noise, and no modal aliasing will occur.

While searching for AFIBFs, the sequence is from high frequency to low frequency (HTL-FS algorithm) or from low frequency to high frequency (LTH-FS algorithm). Since blasting vibration signals are mainly low-frequency signals, this article adopts the LTH-HS algorithm; its specific steps are as follows [17].

Set $i=1, \ldots, M, N_{0}=0$, and $N_{M}=N / 2-1$.

(1) Perform Fourier transform on the original signal $x(t)$, namely, $X[k]=F F T\{x[n]\}$

(2) Set $\mathrm{AFIBF}_{i}=\sum_{k=\left(N_{i-1}+1\right)}^{N_{i}} X[k] \exp j 2 \pi k n / N=a_{i}[n]$ $\exp \left(j \phi_{i}[n]\right)$, and the minimum number of AFIBFs can be obtained; then, set $N_{i-1}+1 \leq N_{i} \leq N / 2-1$, and make its phase $\phi_{i}[n]$ increase monotonically in the interval, $\omega_{i}[n]=\left(\phi_{i}[n+1]-\phi_{i}[n-1] / 2\right) \geq 0, \forall n$

(3) Residual component $r[n]=X[0]+X[N / 2](-1)^{n}$

(4) The instantaneous frequency and amplitude of AFIBFs can be calculated, and the real part of AFIBFs is FIBFs

2.2. The Principle of Interference Component Screening. At present, the selection of trend items of blasting vibration signals generally adopts manual discrimination. The screening of high-frequency noise components mainly depends on the cross-correlation coefficient, which is expressed as

$$
R(i)=\frac{1}{T} \sum_{t=1}^{T} x(t) c_{j}(t)
$$

where $R(i)$ is the cross-correlation coefficient, $T$ is the signal length, $x(t)$ is the original signal, and $c_{j}(t)$ is the modal component obtained by decomposition. $j=1,2, \ldots, J$, $J$ is the number of modes obtained by decomposition. 
The correlation coefficient can indicate the degree of correlation between the two signals. The correlation coefficient between the original signal and the interference term should be 0 ; however, in practice, since the original signal also contains the trend term and noise, the correlation coefficient will not be 0 . Therefore, it is generally believed that if the correlation coefficient between the modal component and the original signal is less than 0.1 , a noise component or a trend component will exist [18].

If the trend item is too large, the decomposed trend item component may account for the main part of the original signal. At this time, the correlation coefficient between the original signal and the trend item component will be larger, so frequency analysis is also introduced to help identify the trend item. Since the blasting vibrometer has an effective frequency collection range, it can be considered an interference item if the collected signal contains parts that exceed the frequency range.

2.3. The Removal of the Trend Item and the Principle of Noise Using FDM. Firstly, the FDM theory is used to decompose the original signal into a series of Fourier intrinsic frequency band functions to obtain the decomposed modal components. Then, the correlation coefficient between the modal component and the original signal is calculated, and the dominant component is selected; at the same time, the trend item and noise component are removed according to the frequency band information. Finally, the screened components are reconstructed to obtain a pure signal. The specific process is shown in Figure 1.

\section{Numerical Simulation Analysis}

3.1. Simulation Signal Establishment. In order to verify the feasibility of FDM removing trend items and noise, the numerical simulation analysis was carried out. At present, the superposition of sine and cosine functions is generally used to simulate the blasting vibration signal [19], and the structure of the analogue signal is as follows:

$$
\left\{\begin{array}{l}
x_{1}=0.2 e^{-15 t} \times \sin (150 \pi t) \\
x_{2}=0.25 \cos (50 \pi t) \\
x_{3}=0.3 \sin (100 \pi t) \times(1+1.5 \sin (25 \pi t)) \\
x=x_{1}+x_{2}+x_{3} \\
z=x+\alpha(t)+\beta(t)
\end{array}\right.
$$

where $x$ is the superposition used to form the original pure signal, $\alpha(t)$ is the artificial noise component, $\beta(t)$ is the artificial trend item, and $z$ is the original signal. The signal waveform diagram is shown in Figure 2.

It can be found that the simulated signal containing noise and trend items has been severely disturbed, and it is difficult to obtain useful information. Therefore, it needs to be preprocessed during signal analysis to remove the interference of trend items and noise.

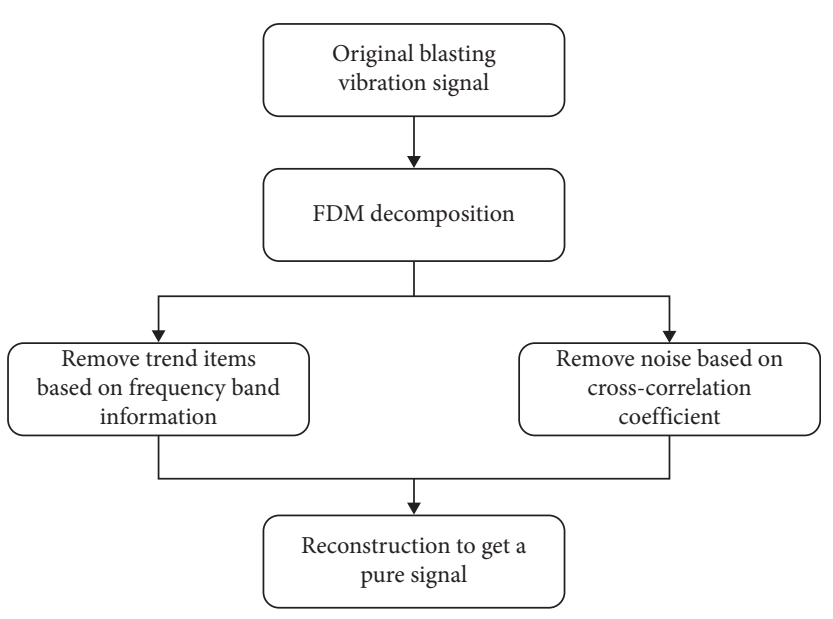

FIgURE 1: Flowchart of noise removal.

3.2. FDM-Based Simulation Signal Trend Item and Noise Removal. FDM decomposition is performed on the simulated signal containing noise and trend items. The frequency is decomposed from low to high, and 40 modal components and 1 residual component $r$ were obtained. Due to space limitations, only five representative low-frequency components and high-frequency components are shown in Figure 3.

The correlation coefficient between the $\mathrm{C} 1$ component and the original signal is 0.8863 . However, by comparing the waveforms in Figure 3, it can be clearly seen that the C1 component is the trend item component. The correlation coefficients between the remaining different modal components and the original signal are calculated and shown in Table 1.

It can be found that the $\mathrm{C} 2-\mathrm{C} 4$ components have a good correlation with the original signal, which are considered to contain useful information in the original signal. In contrast, the correlation coefficients of the remaining components with the original signal are all less than 0.1 , so they can be considered noise components. At the same time, through the analysis of the cross-correlation coefficient, it is found that the cross-correlation coefficient obtained from the useful information component and the noise component is very different, indicating that the useful information and the noise interference in the original signal are effectively separated by FDM. Furthermore, the $\mathrm{C} 2-\mathrm{C} 4$ components were reconstructed to obtain a pure signal, as shown in Figure 4.

3.3. Evaluation of the Removal Effect of the Trend Term and Noise. In order to verify the effect of this method on removing trend items and noise, the original signal was processed with the EMD method, the CEEMDAN method, and the VMD method, respectively; the obtained pure signal is shown in Figure 5.

After analyzing and comparing the waveform diagrams, it can be found that these methods effectively removed the trend item in the original signal, but the denoising effect is obviously different. Among them, the pure signal reconstructed by the EMD method can be found to have obvious 


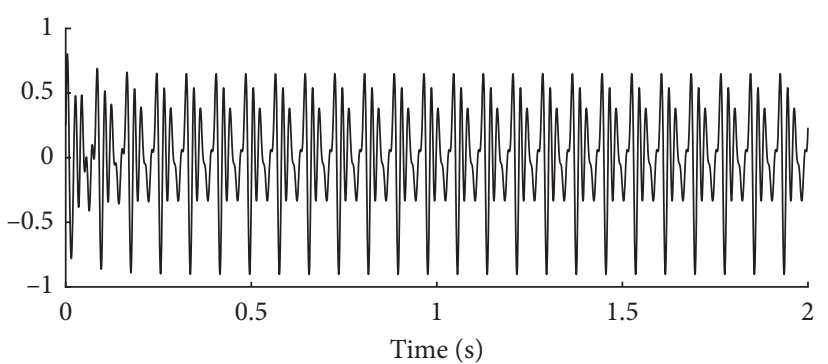

(a)

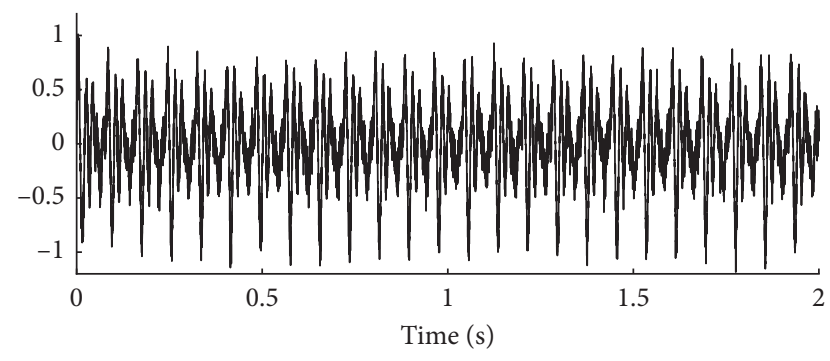

(b)

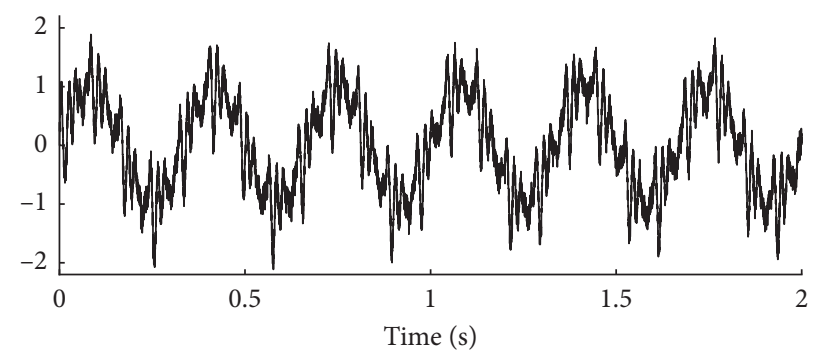

(c)

FIgURE 2: Simulation signal waveform. (a) Original signal. (b) Original signal with noise. (c) Original signal with noise and trend terms.
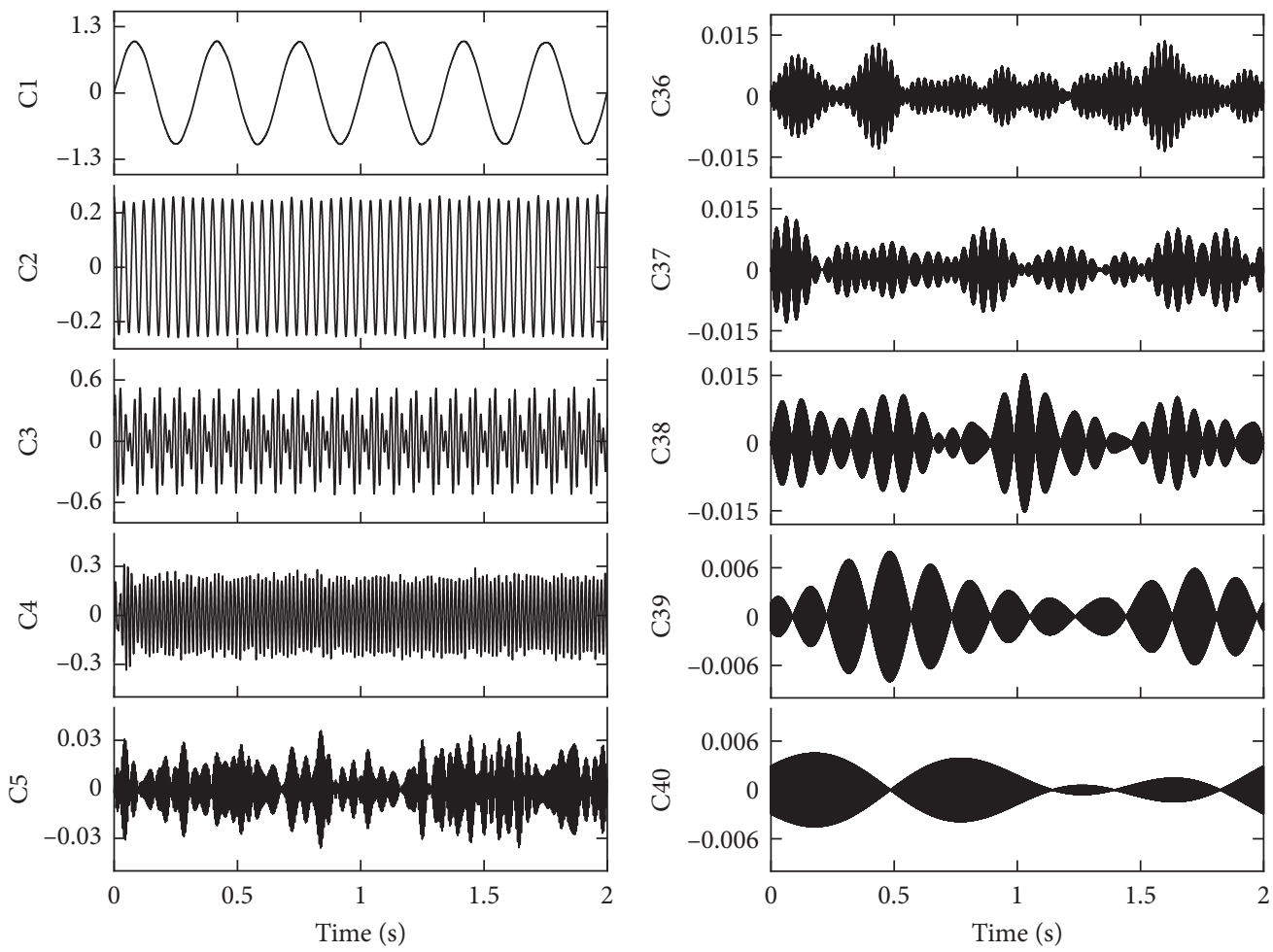

FIgURE 3: Decomposition results of FDM.

glitch noise residue, the CEEMDAN method has slight burr noise residue, and the VMD method has a smoother curve than the first two methods, while its peak part is much lower than the original signal, indicating that useful information in the original signal is removed when the trend item and noise are removed; this is not good for signal analysis. The signal- to-noise ratio (SNR) and root mean square error (RMSE) are further used for quantitative analysis [20]. The higher the signal-to-noise ratio, the smaller the root mean square error, indicating a better denoising effect. The calculation formulas are shown in (5) and (6), and the calculation results are shown in Table 2. 
TABLE 1: The correlation coefficient between the FIBFs and the original signal.

\begin{tabular}{lcccccccc}
\hline FIBFs & C2 & C3 & C4 & C5 & C6 & C7 & C8 & C9 \\
\hline$R$ & 0.2214 & 0.3321 & 0.2025 & 0.0144 & 0.0151 & 0.0192 & 0.0203 & 0.0191 \\
\hline FIBFs & C10 & C11 & C12 & C13 & C14 & C15 & C16 & C17 \\
\hline$R$ & 0.0208 & 0.0273 & 0.0185 & 0.0272 & 0.0308 & 0.0293 & 0.0294 & 0.0285 \\
\hline FIBFs & C18 & C19 & C20 & C21 & C22 & C23 & C24 & C25 \\
\hline$R$ & 0.0250 & 0.0253 & 0.0281 & 0.0289 & 0.0273 & 0.0217 & 0.0186 & 0.0191 \\
\hline FIBFs & C26 & C27 & C28 & C29 & C30 & C31 & C32 & C33 \\
\hline$R$ & 0.0166 & 0.0173 & 0.0149 & 0.0152 & 0.0120 & 0.0113 & 0.0093 & 0.0107 \\
\hline FIBFs & C34 & C35 & C36 & C37 & C38 & C39 & C40 & r \\
\hline$R$ & 0.0088 & 0.0058 & 0.0058 & 0.0070 & 0.0043 & 0.0030 & 0.0006 & 0.0006 \\
\hline
\end{tabular}

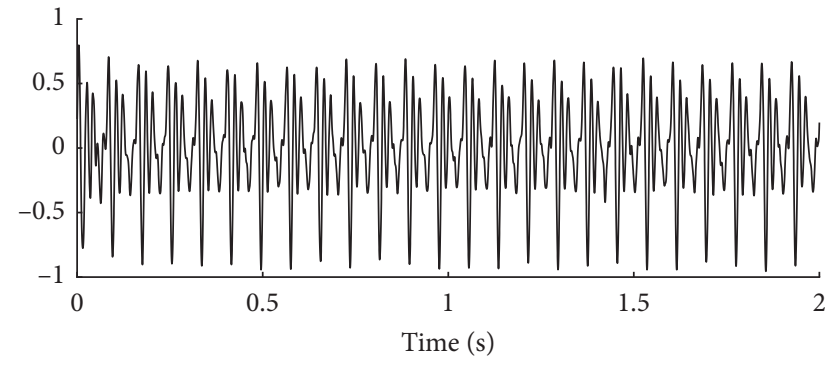

Figure 4: Waveform diagram of the pure signal.

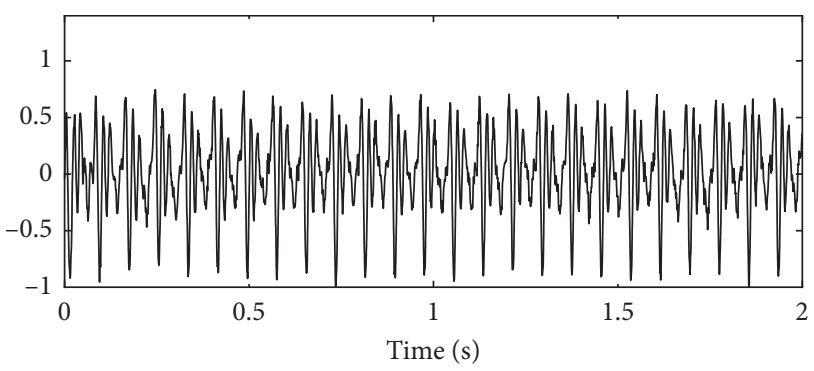

- EMD

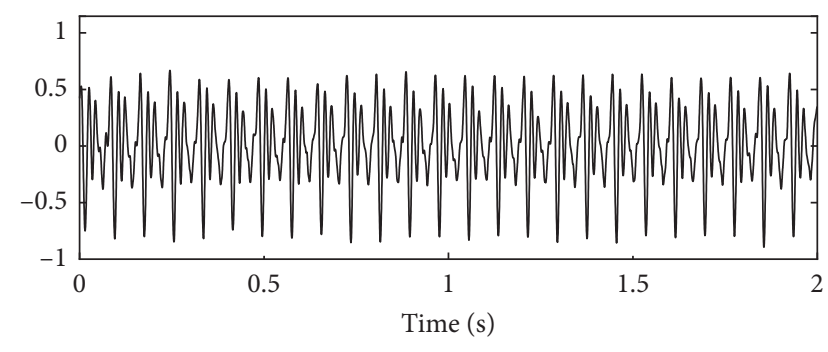

- CEEMDAN

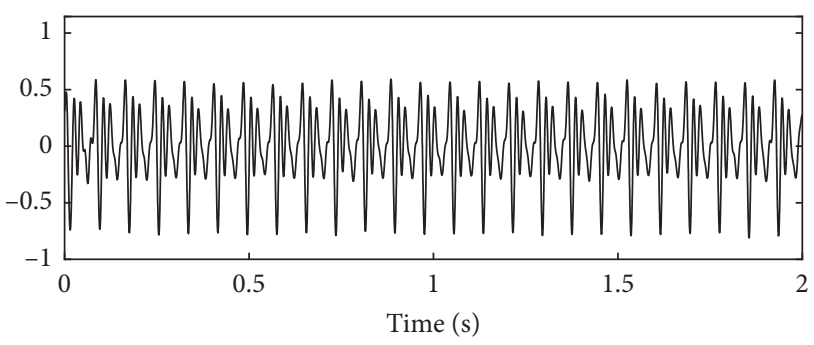

- VMD

FIGURE 5: Comparison of the removal effect of the trend item and noise.

$$
\mathrm{SNR}=10 \times \log _{10}\left\{\frac{\sum_{i=1}^{n} Z_{i}(t)^{2}}{\sum_{i=1}^{n}\left[Z_{i}(t)-Z_{i}^{\prime}(t)\right]^{2}}\right\}
$$

$\mathrm{RMSE}=\sqrt{\frac{1}{n} \sum_{i=1}^{n}}\left[Z_{i}(t)-Z_{i}^{\prime}(t)\right]^{2}$.

After comparing the indicators of the denoising effect of the four methods, it can be found that the pure signal obtained using the VMD method is smoother than that of the CEEMDAN method, but its signal-to-noise ratio is lower, and the root mean square error is higher, indicating that useful information is not effectively retained, which is detrimental to the subsequent signal analysis. The pure signal time history curve obtained using FDM is the smoothest, the signal-to-noise ratio (23.2406) is the highest, and the root mean square error (0.0246) is the smallest, indicating that the useful information of the original signal is retained, and the trend item and noise are effectively removed, which is conducive to the accurate analysis of the subsequent signal.

\section{The Analysis of the Measured Signal}

4.1. The Acquisition of the Measured Signal. This blasting vibration signal comes from a tunnel blasting project. The blasting parameters are shown in Table 3, and the blasting vibration signal is shown in Figure 6.

It can be seen from Figure 6 that the blasting vibration signal obviously contains trend items and high-frequency noise, so it needs to be preprocessed to facilitate subsequent signal analysis.

4.2. FDM-Based Trend Item and Noise Removal. The original signal is decomposed by FDM; from low to high frequency, 43 modal components and 1 residual component are obtained. Due to space limitations, only five representative modal components in the low-frequency part and modal components in the high-frequency part are, respectively, shown in Figure 7 . At the same time, the dominant frequency of the modal component and its correlation with the original signal are calculated and shown in Table 4. 
TABLE 2: Comparison of noise reduction effects.

\begin{tabular}{lcccc}
\hline Method & EMD & CEEMDAN & VMD & \\
\hline SNR & 15.4870 & 17.8109 & 15.8413 & 23.2406 \\
RMSE & 0.06 & 0.0459 & 0.0576 & 0.0246 \\
\hline
\end{tabular}

TABLE 3: Blasting parameters.

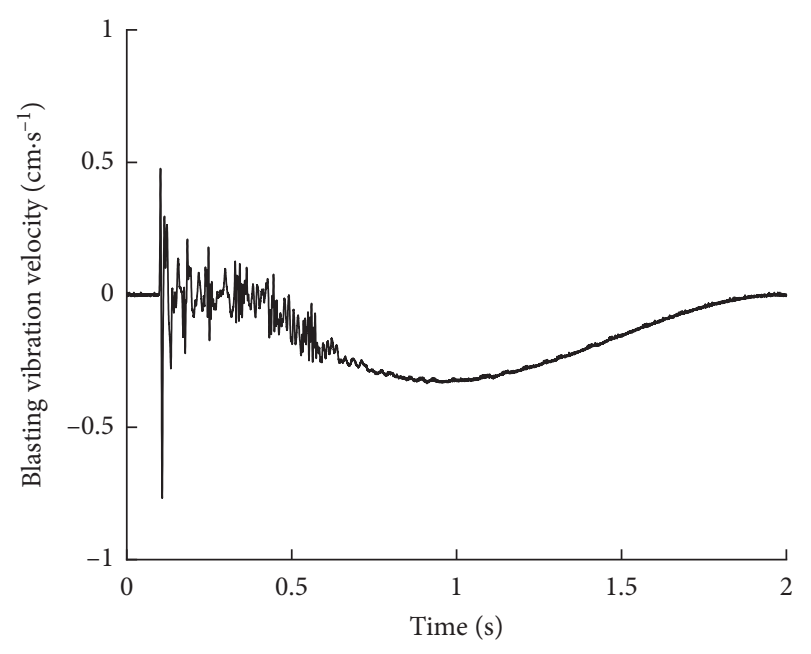

FIgURE 6: Blasting vibration signal waveform.
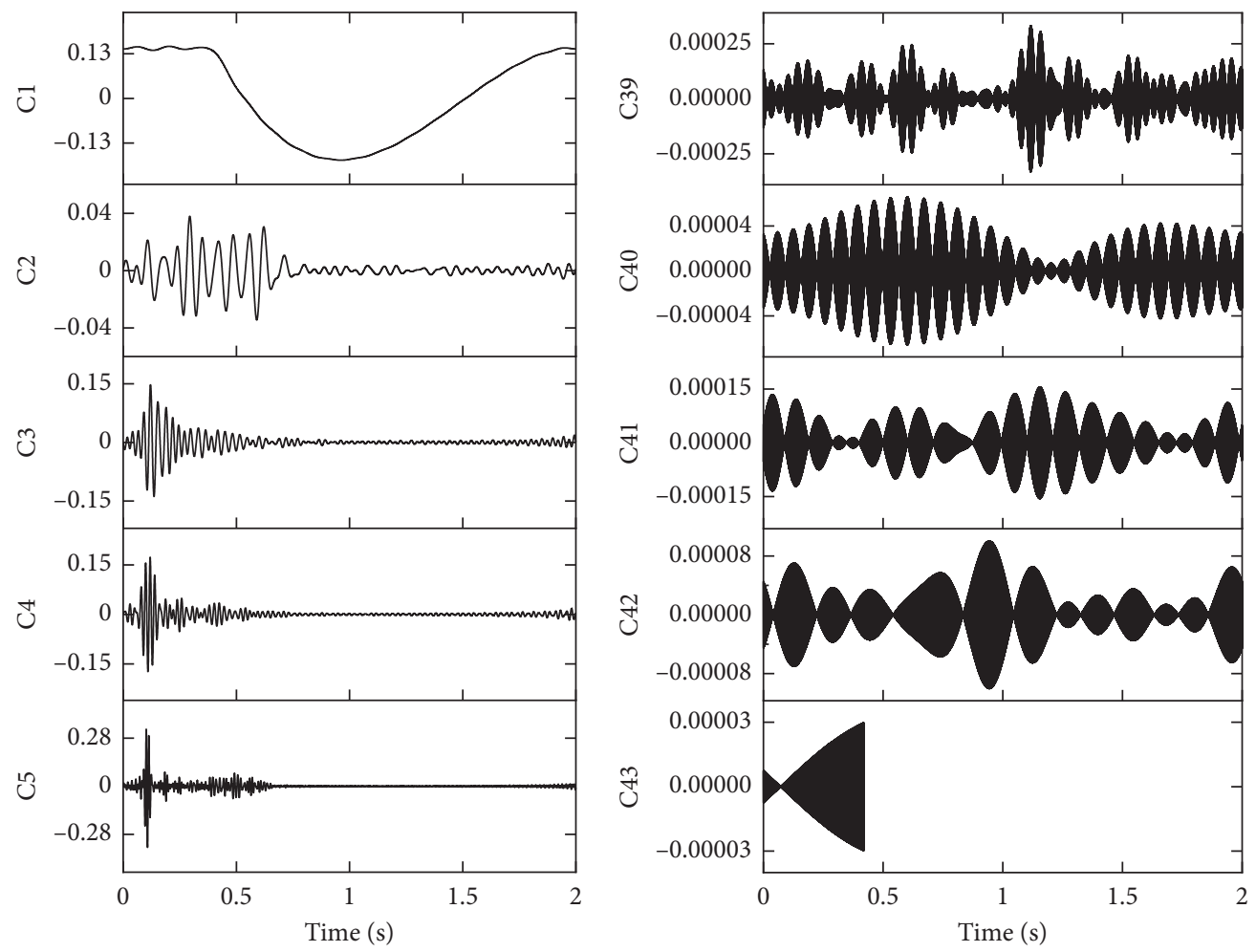

Figure 7: Decomposition results of FDM. 
TABLE 4: Related parameters of FIBFs.

\begin{tabular}{lccccccccc}
\hline FIBFs & C1 & C2 & C3 & C4 & C5 & C6 & C7 & C8 & C9 \\
\hline$R$ & 0.9336 & 0.1986 & 0.4685 & 0.4573 & 0.6024 & 0.2956 & 0.1400 & 0.2165 & 0.1144 \\
\hline Main frequency $(\mathrm{Hz})$ & 2.0 & 6.2 & 13.4 & 17.0 & 30.6 & 44.8 & 69.2 & 79.4 & 87.6 \\
\hline FIBFs & $\mathrm{C} 10$ & $\mathrm{C} 11$ & $\mathrm{C} 12$ & $\mathrm{C} 13$ & $\mathrm{C} 14$ & $\mathrm{C} 15$ & $\mathrm{C} 16$ & $\mathrm{C} 17$ & $\mathrm{C} 18$ \\
\hline$R$ & 0.0131 & 0.0073 & 0.0069 & 0.0070 & 0.0069 & 0.0076 & 0.0081 & 0.0110 & 0.0125 \\
\hline Main frequency $(\mathrm{Hz})$ & 126.0 & 150.4 & 179.4 & 190.8 & 223.6 & 256.0 & 288.2 & 316.6 & 351.0 \\
\hline FIBFs & $\mathrm{C} 19$ & $\mathrm{C} 20$ & $\mathrm{C} 21$ & $\mathrm{C} 22$ & $\mathrm{C} 23$ & $\mathrm{C} 24$ & $\mathrm{C} 25$ & $\mathrm{C} 26$ & $\mathrm{C} 27$ \\
\hline$R$ & 0.0182 & 0.0152 & 0.0107 & 0.0098 & 0.0089 & 0.0087 & 0.0097 & 0.0089 & 0.0084 \\
\hline Main frequency $(\mathrm{Hz})$ & 423.6 & 431.2 & 537.2 & 586.0 & 652.0 & 683.6 & 733.4 & 783.8 & 842.4 \\
\hline FIBFs & $\mathrm{C} 28$ & $\mathrm{C} 29$ & $\mathrm{C} 30$ & $\mathrm{C} 31$ & $\mathrm{C} 32$ & $\mathrm{C} 33$ & $\mathrm{C} 34$ & $\mathrm{C} 35$ & $\mathrm{C} 36$ \\
\hline$R$ & 0.0068 & 0.0057 & 0.0065 & 0.0049 & 0.0038 & 0.0047 & 0.0047 & 0.0029 & 0.0036 \\
Main frequency $(\mathrm{Hz})$ & 852.0 & 886.4 & 908.8 & 935.4 & 945.6 & 947.0 & 964.4 & 971.0 & 978.8 \\
\hline FIBFs & $\mathrm{C} 37$ & $\mathrm{C} 38$ & $\mathrm{C} 39$ & $\mathrm{C} 40$ & $\mathrm{C} 41$ & $\mathrm{C} 42$ & $\mathrm{C} 43$ & $r$ \\
\hline$R$ & 0.0027 & 0.0023 & 0.0021 & 0.0007 & 0.0014 & 0.0008 & 0.0005 & 0.0005 & \\
\hline Main frequency $(\mathrm{Hz})$ & 988.2 & 990.4 & 996.6 & 997.0 & 998.4 & 999.0 & 999.8 & 0
\end{tabular}

The minimum measurable frequency of the blasting vibrometer used is $5 \mathrm{~Hz}$, and the part below $5 \mathrm{~Hz}$ is regarded as the trend item. It can be seen from Table 4 that although the correlation coefficient between the $\mathrm{C} 1$ component and the original signal is 0.9336 , its main frequency is $2 \mathrm{~Hz}$, which is an obvious trend item component. This is because the trend item has a low frequency, leading to a greater impact on the original signal. When the cross-correlation coefficient is less than 0.1 , the component is the noise component. It can be found that when the frequency is higher than $120 \mathrm{~Hz}$, the correlation coefficient between the modal component and the original signal suddenly changes from higher than 0.1 to 0.0131 , indicating that the noise and useful information are effectively separated, further verifying the excellent performance of FDM in noise removal of blasting vibration signals.

In summary, according to the frequency information and the cross-correlation coefficient, the $\mathrm{C} 1$ component is considered the trend component, and the C10-C43 components are the high-frequency noise components, which should be removed. C2-C9 components are selected to reconstruct the pure signal, as shown in Figure 8.

\subsection{Evaluation of Trend Item and Noise Removal Effect.} In order to evaluate the removal effect of the trend item and noise in the measured signal, the original signal was processed by the EMD method, the CEEMDAN method, and the VMD method, respectively, and the comparison and analysis were performed to obtain the pure signal as shown in Figure 9.

It can be found that all these methods can effectively remove the trend item in the original signal; among them, the EMD method has more residual burr noise, and the peak vibration speed is reduced; although the CEEMDAN method and VMD method have better noise removal effects, their peak vibration velocity is also reduced to a certain extent, which is unfavourable for signal analysis and has an adverse effect on optimizing blasting parameters and controlling blasting hazards. The effect of these methods is evaluated from three aspects, including signal-to-noise ratio,

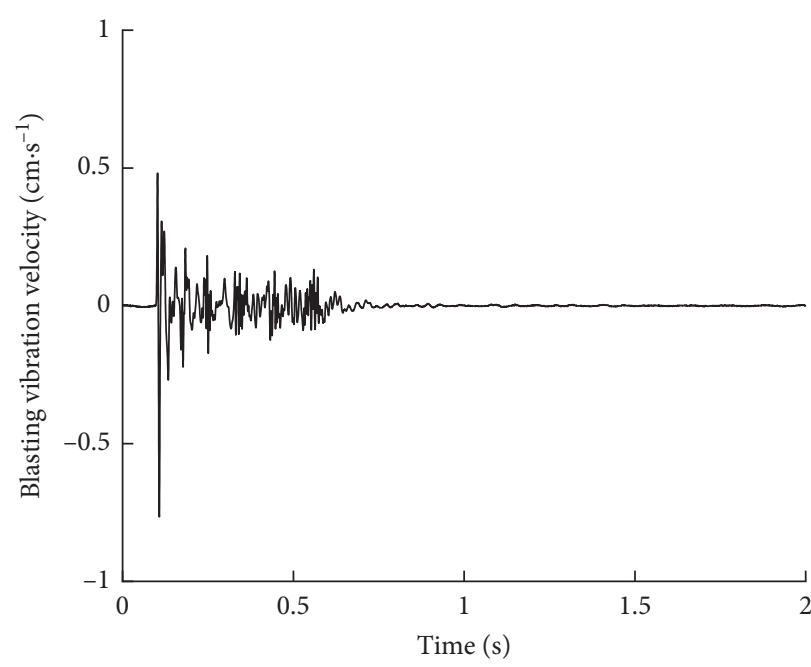

Figure 8: Pure signal.

root mean square error, and peak vibration velocity. The calculation results are shown in Table 5 .

The peak vibration velocity of the original signal is $0.7673 \mathrm{~cm} / \mathrm{s}$. According to the data in Table 5, it can be found that although the signal waveform is the smoothest after processing by the VMD method, the signal-to-noise ratio is the lowest, and the peak vibration velocity is also significantly reduced, which means the VMD method will remove the useful information while erasing noise, which is not good for accurate signal analysis. Compared with the EMD method and the VMD method, the signal-tonoise ratio of the CEEMDAN method is significantly improved, and the peak vibration velocity is closer to reality. The pure signal obtained by FDM has the highest signal-to-noise ratio (28.3736), the root mean square error (0.0018) is the smallest, and the peak vibration velocity is closest to the actual value. It shows that while using the FDM to remove the interference of trend items and noise, the useful information in the original signal will be retained as much as possible, and furthermore, 

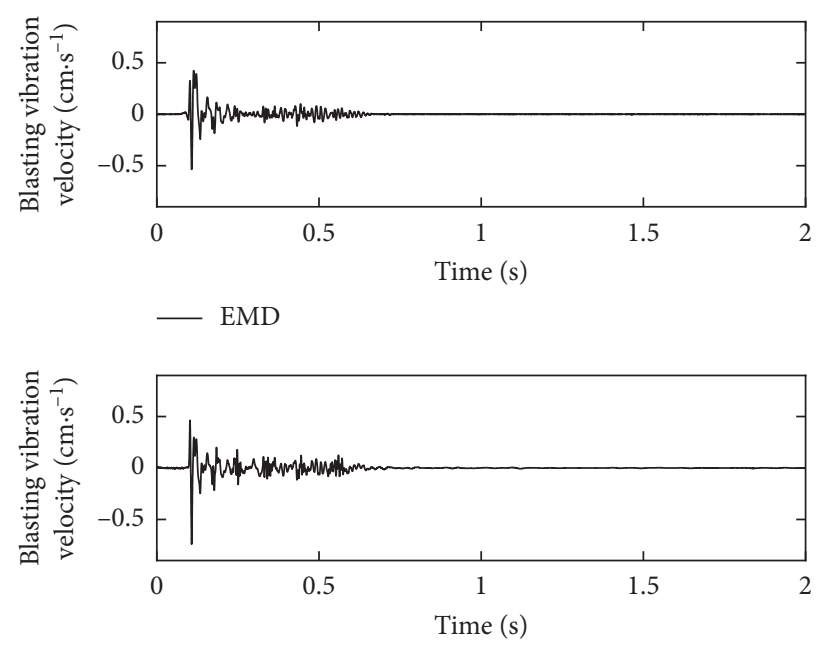

- CEEMDAN

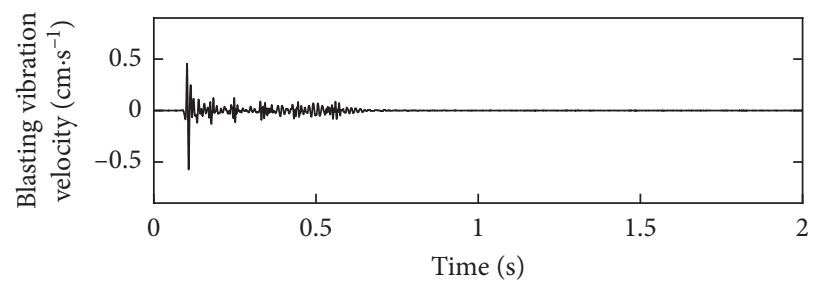

VMD

Figure 9: Comparison of the effects of different methods.

TABLE 5: Comparison of the effects of different methods.

\begin{tabular}{lccc}
\hline Method & SNR & RMSE & Peak vibration velocity $(\mathrm{cm} / \mathrm{s})$ \\
\hline EMD & 6.3379 & 0.0229 & 0.5364 \\
CEEMDAN & 19.2246 & 0.0052 & 0.7409 \\
VMD & 6.1100 & 0.0235 & 0.5735 \\
FDM & 28.3736 & 0.0018 & 0.7658 \\
\hline
\end{tabular}

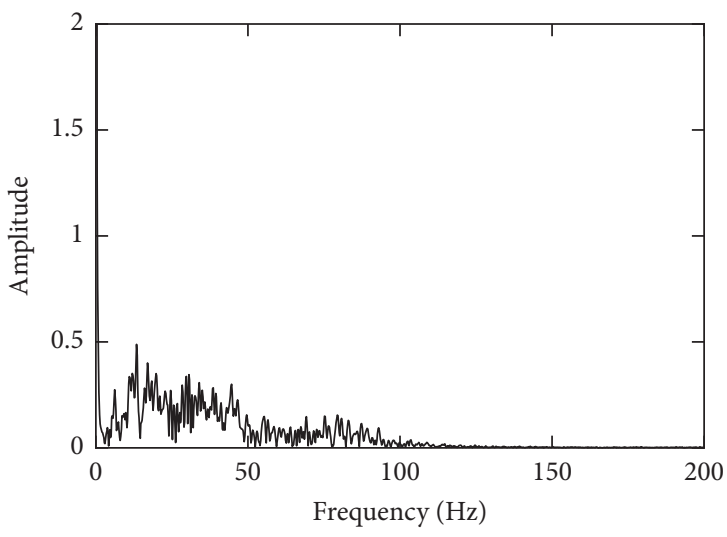

(a)

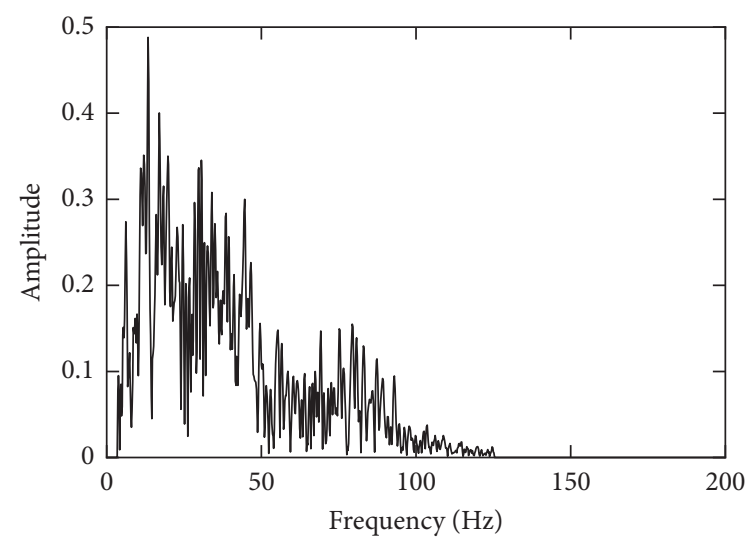

(b)

FIgURE 10: Signal spectrogram. (a) Original signal spectrogram. (b) Pure signal spectrogram.

spectrum analysis is performed to verify the effect. The original signal spectrogram and the obtained pure signal spectrogram after FDM processing are compared in Figure 10.
It can be seen that when the trend item is not excluded, the main frequency of the original signal is around $2 \mathrm{~Hz}$, and it contains much high-frequency noise. The main frequency of the pure signal after FDM processing returns to the normal 
range $(5-100 \mathrm{~Hz})$ and is basically consistent with the original signal's spectrogram in this range, indicating that the detailed features in the original signal can be effectively retained, and the high-frequency noise is effectively eliminated.

\section{Results and Discussion}

Based on a tunnel blasting project, this paper introduces an FDM-based method of removing the blasting vibration signal trend item and noise. By analyzing the measured blasting vibration signals, the following conclusions can be drawn:

(1) Using FDM to decompose signals containing trend items and noise can effectively separate low-frequency trend items, high-frequency noise, and useful signal information, further facilitating the extraction of useful signal information.

(2) After FDM decomposition, the pure signal is reconstructed by the dominant components obtained by screening the frequency information and the cross-correlation coefficient, which can effectively retain the detailed features in the original signal, and the frequency characteristics are basically the same.

(3) Compared with common methods, when the blasting vibration signal contains both trend items and high-frequency noise, the pure signal obtained by FDM processing has the best effect. The signal-tonoise ratio (28.3736) is the highest, the root mean square error (0.0018) is the smallest, and the peak vibration velocity $(0.7658 \mathrm{~cm} / \mathrm{s})$ is closest to the actual value $(0.7673 \mathrm{~cm} / \mathrm{s})$.

\section{Data Availability}

The data used to support the findings of this study are included within the article.

\section{Conflicts of Interest}

The authors declare that they have no conflicts of interest to this work.

\section{Acknowledgments}

This study was financially supported by the National Natural Science Foundation of China (no. 51878242), the Natural Science Foundation of Hebei Province (E2020404007), the Graduate Student Innovation Fund of Hebei Province (CXZZSS2021153), and the Innovation Fund of Hebei University of Architecture (XY202114), and the support is gratefully appreciated.

\section{References}

[1] H. Yuan, X. Liu, and Y. Bian, "Analysis of acoustic wave frequency spectrum characters of rock mass under blasting damage based on the HHT method," Advances in Civil Engineering, vol. 2018, no. 4, 8 pages, Article ID 9207476, 2018.
[2] Z. Wu and N. E. Huang, "Ensemble empirical mode decomposition: a noise-assisted data analysis method," Advances in Adaptive Data Analysis, vol. 1, no. 1, pp. 1-41, 2009.

[3] L. J. Fu, F. S. Wang, and Z. N. Liu, "Application of improved adaptive CEEMD method in denoising of ECG signal," Journal of Electronic Measurement and Instrument, vol. 34, no. 4, pp. 50-57, 2020.

[4] A. R. Hassan, A. Subasi, and Y. C. Zhang, "Epilepsy seizure detection using complete ensemble empirical mode decomposition with adaptive noise," Knowledge-Based Systems, vol. 191, no. 5, pp. 1-12, 2020.

[5] P. Chen and X. Q. Zhao, "Feature extraction of early faults of rolling bearings based on optimized VMD and improved threshold noise reduction," Journal of Vibration and Shock, vol. 40, no. 13, pp. 146-153, 2021.

[6] C. Li, S. F. Liang, C. P. Liu, J. Cheng, and D. S. Liu, "Elimination method of trend term of blasting vibration signal based on improved ensemble empirical mode decomposition," Transactions of Beijing Institute of Technology, vol. 41, no. 6, pp. 636-641, 2021.

[7] B. Jia, T. L. Ling, S. J. Hou, D. Liu, and X. Wang, "Application of variable mode decomposition in the removal of blasting signal trend items," Explosion and Shock Waves, vol. 40, no. 4, pp. 123-131, 2020.

[8] L. Zhang, T. H. Ling, Z. H. Chen, S. Zhang, and B. Yu, "Determining the actual delay time of millisecond blasting based on the improved CEEMDAN method," Journal of Vibration and Shock, vol. 39, no. 20, pp. 274-280, 2020.

[9] J. Huang, J. Gao, and L. Zhang, "Study on feature signal extraction method of axlebox bearing fault based on wavelet denoising and HHT transformation," Machine Tool \& Hydraulics, vol. 48, no. 10, pp. 50-55, 2020.

[10] R. L. Shan, Y. W. Song, Y. Bai, L. Zhang, T. Zhou, and S. Zhang, "Research on the energy attenuation characteristics of blasting vibration signals based on wavelet packet transformation," Journal of Mining Science and Technology, vol. 3, no. 2, pp. 119-128, 2018.

[11] Q. Y. Li, H. W. Wang, J. B. Wang, H. D. Zeng, and J. Zheng, "Research on preprocessing of blasting vibration signal based on EEMD and wavelet threshold," Mining and Metallurgical Engineering, vol. 41, no. 3, pp. 28-31+36, 2021.

[12] X. Q. Fu, R. S. Yang, J. F. Liu, H. Zhang, and R. Zhang, "Baseline drift correction and de-noising method of shaft lining vibration signal in near field of freezing vertical shaft blasting," Explosion and Shock Waves, vol. 40, no. 9, pp. 100-112, 2020.

[13] Y. Liu, X. B. Liu, and S. Liang, "Aeroengine rotor fault diagnosis based on fourier decomposition method," China Mechanical Engineering, vol. 30, no. 18, pp. 2156-2163, 2019.

[14] Z. M. Jin, L. P. Sun, G. You, S. Lv, and C. Yi, "Improved Fourier decomposition and its application to fault diagnosis of rolling bearing," Machine Tool \& Hydraulics, vol. 49, no. 6, pp. 163-169, 2021.

[15] X. W. Fu and X. Q. Gao, "Rolling bearing fault diagnosis based on FDM and singular value difference spectrum," Acta Metrologica Sinica, vol. 39, no. 5, pp. 688-692, 2018.

[16] P. Singh, S. D. Joshi, R. K. Patney, and K Saha, "The fourier decomposition method for nonlinear and non-stationary time series analysis," Proceedings. Mathematical, physical, and engineering sciences, vol. 473, no. 2199, pp. 2016087120160914, 2017.

[17] G. R. Zhang, X. Y. Wang, and W. B. Guo, "Fault diagnosis method of rolling bearing based on fourier decomposition method and 1.5-dimensional teager energy spectrum," 
Journal of Mechanical Transmission, vol. 41, no. 3, pp. 191196, 2017.

[18] H. L. Fei, M. Liu, G. J. Qu, and Y. Gao, "Denoising method of blasting vibration signal based on ensemble empirical mode decomposition-wavelet threshold method," Explosion and Shock Waves, vol. 38, no. 1, pp. 112-118, 2018.

[19] W. H. Yi, L. S. Liu, L. Yan, and B. Dong, "Vibration signal denoising based on improved EMD algorithm," Explosion and Shock Waves, vol. 40, no. 9, pp. 77-87, 2020.

[20] X. L. Zhou, X. L. Xu, Y. Wang, W. Liu, Z. Jiang, and F. Ma, “A gear signal de-noising method based on variational mode decomposition and maximal overlap discrete wavelet packet transform," Journal of Vibration and Shock, vol. 40, no. 12, pp. $265-274+289,2021$. 